\title{
Stationary Solutions for Nonlinear Schrödinger Equations by Lie Group Analysis
}

\author{
S.T. RAZA Rizvi ${ }^{a, *}$, I. AfZAL ${ }^{a}, \mathrm{~K}$ Ali $^{a}$ AND M. Younis ${ }^{b}$ \\ ${ }^{a}$ Department of Mathematics, COMSATS University Islamabad, Lahore Campus, Pakistan \\ ${ }^{b}$ Department of Mathematics and Statistics, The University of Lahore, Lahore, Pakistan \\ (Received January 17, 2019; revised version May 17, 2019; in final form May 19, 2019)
}

\begin{abstract}
In this paper we obtain the stationary solutions of various nonlinear Schrödinger equations. We use Lie symmetry method to find the stationary solutions of the Gerdjikov-Ivanov equation, cubic-quintic and paraxial nonlinear Schrödinger equations.
\end{abstract}

DOI: 10.12693/APhysPolA.136.187

PACS/topics: stationary solutions, optical solitons, Lie symmetry

\section{Introduction}

The theory of nonlinear Schrödinger equations (NLSE) plays a vital role in various areas of physical, biological, and engineering sciences. The governing NLSE shows up in distinctive fields, including fluid dynamics, nonlinear optics and plasma physics. A lot of work has been done to find soliton solutions for various forms of NLSEs [1-20]. Eslami and Neirameh studied the exact soliton solutions for higher order NLSE [13]. Biswas found the bright and dark soliton solution in optical fiber under parabolic law of non-linearity [12]. Yang et al. studied fourth order variable coefficient NLSE for obtaining bright soliton interaction [1]. Aouadi et al. obtained $W$-shaped, dark, and bright soliton for Biswas-Arshed equation [7]. Zhou et al. studied Triki-Biswas equation for chirped singualr solitons [8]. Zhou et al. also obtained optical solitons for unstable NLSE [9]. Inc et al. obtained the optical solitons and modulation instability analysis of an integrable model of $(2+1)$-Dimensional Heisenberg ferromagnetic spin chain equation [15]. Khalique and Biswas found the solution of NLSE with non-Kerr law non-linearity [17]. Ekici et al. found the optical solitons with DWDM technology and four-wave mixing [14]. Zhang et al. studied the interactions of vector antidark solitons for the coupled nonlinear Schrödinger equation in inhomogeneous fibers [19]. Biswas and Khalique found the stationary solutions for the nonlinear dispersive Schrödinger equation (NDSE) which is an important generalized form of NLSE [10] and later on they also obtained the stationary solutions for NDSE with generalized evolution [11]. Here, we will find the stationary solutions of three important NLSEs, namely, GerdjikovIvanov equation, cubic-quintic equation, and paraxial equation.

*corresponding author; e-mail: srizvi@cuilahore.edu.pk

\section{Gerdjikov-Ivanov equation}

One of the famous NLSE is Gerdjikov-Ivanov (GI) equation [18] having dimensionless form as

$$
\mathrm{i} q_{t}+a q_{x x}+\mathrm{i} b q^{2} q_{x}^{*}+c|q|^{4} q=0 \text {. }
$$

We consider the localized stationary solution to Eq. (1) of the form

$$
q(x, t)=\phi(x) \mathrm{e}^{\mathrm{i} \lambda t} .
$$

Using Eq. (2) along with its derivatives into Eq. (1), we get the real and imaginary parts, the real part is

$$
\lambda \phi-a \phi^{\prime \prime}+c \phi^{5}=0,
$$

and the imaginary parts gives us $b=0$. Equation (3) has a single lie point symmetry, let us say $X=\partial / \partial x$. It can be seen that the two invariants are

$$
\begin{aligned}
& u=\phi, \\
& v=\phi^{\prime} .
\end{aligned}
$$

Considering $u$ as the independent variable and $v$ as the dependent variable, (3) can be rewritten as

$$
\frac{\mathrm{d} v}{\mathrm{~d} u}=\frac{\lambda u}{a v}+\frac{c u^{5}}{a v} .
$$

Solving this differential equation and rewriting in terms of $\phi$ leads to

$$
\frac{\mathrm{d} \phi}{\mathrm{d} x}=\sqrt{\frac{c \phi^{6}+3 \phi^{2}+6 a c_{1}}{3 a}} .
$$

The resulting quadrature is

$$
x+c_{2}=\int \sqrt{\frac{3 a}{c \phi^{6}+3 \phi^{2} \lambda+6 a c_{1}}} \mathrm{~d} \phi .
$$

The integral in (8) can be evaluated on choosing the arbitrary constant $c_{1}$ to be zero. In that case, (8) becomes

$$
x+c_{2}=\frac{\sqrt{a}}{2 \sqrt{\lambda}}\left[\ln \left(\phi^{2}\right)-\ln \left(3 \lambda+\sqrt{9 \lambda^{2}+3 c \lambda \phi^{4}}\right)\right] .
$$

The 3D, 2D surfaces and the density plot for Eq. (30) have been formed in Fig. 1 by considering $a=\lambda=1$. 

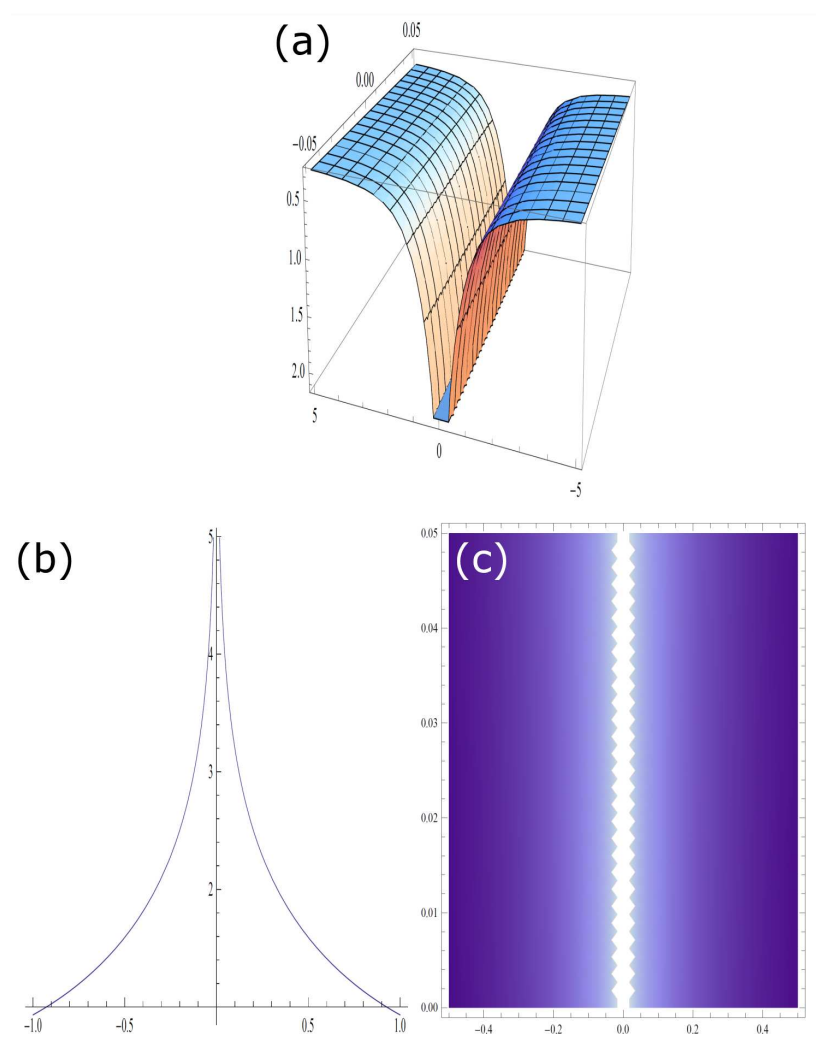

Fig. 1. (a) 3D graph, (b) 2D graph, (c) density graph.

\section{Cubic-quintic equation}

The dimensionless form of cubic-quintic equation is given by [16]:

$$
\mathrm{i} q_{t}+a q_{x x}+\left(b_{1}|q|^{-4}+b_{2}|q|^{2}+b_{3}|q|^{4}\right) q=0 .
$$

We consider the localized stationary solution to Eq. (10) of the form

$$
q(x, t)=\phi(x) \mathrm{e}^{\mathrm{i} \lambda t} .
$$

where $\lambda$ is a constant and the function $\phi$ depends on the variable $x$ alone. Thus $\phi(x)$ satisfies the time independent inhomogeneous nonlinear ordinary differential equation (ODE) that is given by

$$
\lambda \phi-a \phi^{\prime \prime}-b_{1} \phi^{-3}-b_{2} \phi^{3}+b_{3} \phi^{5}=0
$$

Equation (12) has a single Lie point symmetry, which can be taken as $X=\partial / \partial x$. It can be easily seen that the two invariants are

$$
\begin{aligned}
& u=\phi \\
& v=\phi^{\prime}
\end{aligned}
$$

Considering $u$ as the independent variable and $v$ as the dependent variable, (12) can be rewritten as

$$
\frac{\mathrm{d} v}{\mathrm{~d} u}=\frac{1}{a v}\left[\lambda u-b_{1} u^{-3}-b_{2} u^{3}-b_{3} u^{5}\right] .
$$

Solving this differential equation and rewriting in terms of the variable $\phi$ gives us

$$
\frac{\mathrm{d} \phi}{\mathrm{d} x}=\sqrt{\frac{6 b_{1} \phi^{-2}-3 b_{2} \phi^{4}-2 b_{3} \phi^{6}+6 \phi^{2} \lambda+12 a c_{1}}{6 a}} .
$$

The resulting quadrature is

$$
\begin{aligned}
& x+c_{2}= \\
& \quad \int \sqrt{\frac{6 a}{6 b_{1} \phi^{-2}-3 b_{2} \phi^{4}-2 b_{3} \phi^{6}+6 \phi^{2} \lambda+12 a c_{1}}} \mathrm{~d} \phi,
\end{aligned}
$$

where $c_{1}$ and $c_{2}$ are arbitrary constants of integration. Thus, Eq. (17) represents the stationary solution to cubic-quintic equation given by (10).

\section{Paraxial equation}

The dimensionless form of paraxial NLSE [20] is given by

$$
\mathrm{i} u_{z}+\frac{\alpha}{2} u_{t t}+\frac{\beta}{2} u_{y y}+\gamma|u|^{2} u=0 .
$$

We consider that the localized stationary solution to Eq. (18) is

$$
u(y, z, t)=\phi(\xi) \mathrm{e}^{\mathrm{i} a \xi},
$$

where

$$
\xi=y+z-c t .
$$

In Eq. (19) $a$ is a constant and $\phi$ only depends on $\xi$, thus $\phi$ satisfies the following inhomogeneous nonlinear ODE:

$$
\begin{gathered}
\mathrm{i}\left(\phi^{\prime}+\mathrm{i} a \phi\right)+\frac{\alpha}{2}\left(c^{2} \phi^{\prime \prime}+2 \mathrm{i} a c^{2} \phi^{\prime}-a^{2} c^{2} \phi\right) \\
+\frac{\beta}{2}\left(\phi^{\prime \prime}+2 \mathrm{i} a \phi^{\prime}-a^{2} \phi\right)+\gamma \phi^{3}=0 .
\end{gathered}
$$

For simplification we will separate the real and imaginary parts. Real part of Eq. (21) is

$$
-\left(\frac{\alpha}{2} a c^{2}+\frac{\beta}{2} a+1\right) a \phi+\left(\frac{\alpha}{2} c^{2}+\frac{\beta}{2}\right) \phi^{\prime \prime}+\gamma \phi^{3}=0,
$$

and the imaginary part is

$$
\left(1+\alpha a c^{2}+\beta a\right) \phi^{\prime}=0,
$$

where $\phi^{\prime} \neq 0$, which means

$$
1+\alpha a c^{2}+\beta a=0 \text {. }
$$

Then Eq. (22) becomes

$$
\phi^{\prime \prime}+a^{2} \phi-2 a \gamma \phi^{3}=0 .
$$

Equation (25) has a single Lie point symmetry, which can be taken as $X=\partial / \partial x$. It can be easily seen that the two invariants are

$$
\begin{aligned}
& u=\phi \\
& v=\phi^{\prime} .
\end{aligned}
$$

Considering $u$ as the independent variable and $v$ as the dependent variable, (25) can be rewritten as

$$
\frac{\mathrm{d} v}{\mathrm{~d} u}=\left(2 a \gamma u^{3}-a^{2} u\right) v^{-1} .
$$

Solving this differential equation and rewriting in terms of the variable $\phi$ gives us

$$
\frac{\mathrm{d} \phi}{\mathrm{d} \xi}=\sqrt{-a^{2} \phi^{2}+a \phi^{4}+2 c_{1}} .
$$


By putting $c_{1}=0$, the resulting quadrature is

$$
\begin{aligned}
& \xi+c_{2}=\frac{1}{a}\left(\log (\phi)-\log \left(a-\sqrt{a\left(a-u^{2}\right)}\right)\right) \\
& \times \sqrt{\frac{a-\phi^{2}}{-a+\phi^{2}}} .
\end{aligned}
$$

The 3D, 2D surfaces and the contour plot for Eq. (30) have been formed in Fig. 2 by considering $a=1$.

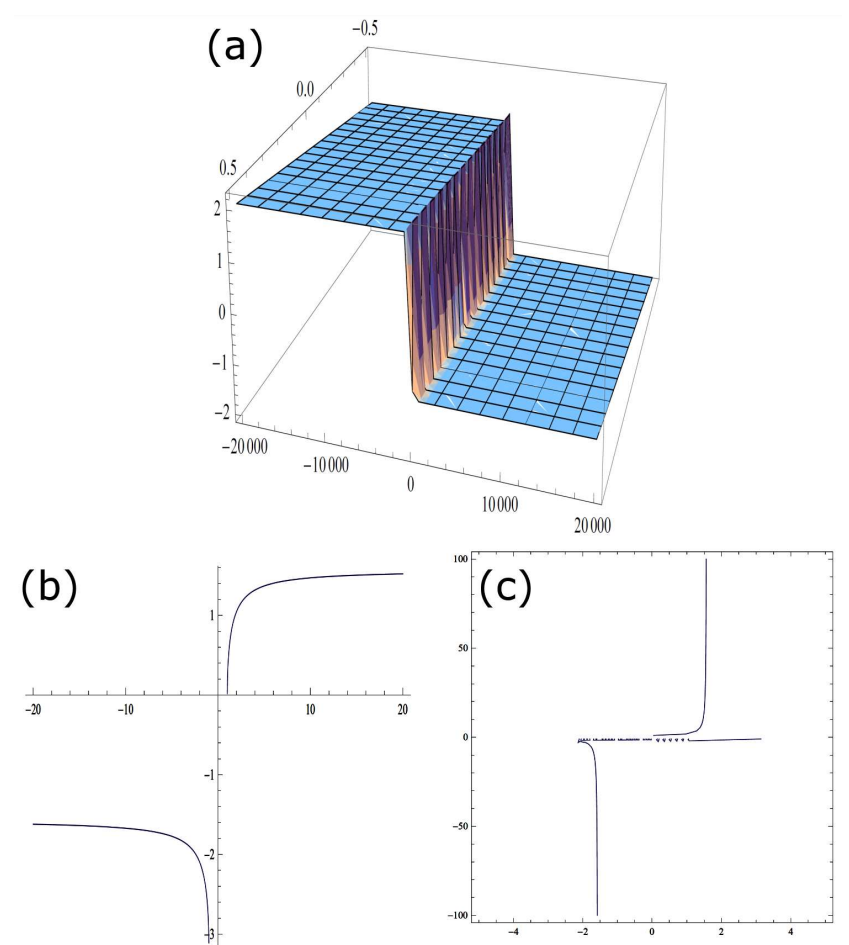

Fig. 2. (a) 3D graph, (b) 2D graph, (c) contour plot.

\section{Conclusion}

In this paper, we obtained stationary solutions for the Gerdjikov-Ivanov equation, cubic-quintic NLSE and paraxial NLSE. The stationary solutions are used in different areas of physics like plasma physics, nonlinear optics, fluid dynamics, and many more. The solutions appear in form of Appell's hypergeometric function and look non-trivial. By using the same technique of Biswas and Khalique for finding the stationary solutions for the nonlinear dispersive Schrödinger equation (NDSE) [10], we obtained the stationary solutions for above mentioned NLSEs. All the results presented in this paper are new and may be useful in optical fiber industry.

\section{References}

[1] C. Yang, Q. Zhou, H. Triki, M. Mirzazadeh, M. Ekici, W. Liu, A. Biswas, M. Belic, Nonlin. Dynam. 95, 983 (2019).

[2] C. Yang, W. Liu, Q. Zhou, D. Mihalache, B.A. Malomed, Nonlin. Dynam. 95, 369 (2019).

[3] X. Liu, H. Triki, Q. Zhou, M. Mirzazadeh, W. Liu, A. Biswas, M. Belic, Nonlin. Dynam. 95, 143 (2019).

[4] W. Yu, Q. Zhou, M. Mirzazadeh, W. Liu, A. Biswas, J. Adv. Res. 15, 69 (2019).

[5] C. Yang, A.M. Wazwaz, Q. Zhou, W. Liu, Laser Phys. 29, 035401 (2019).

[6] X. Liu, H. Triki, Q. Zhou, W. Liu, A. Biswas, Nonlin. Dynam. 94, 703 (2018).

[7] S. Aouadi, A. Bouzida, A.K. Daoui, H. Triki, Q. Zhou, S. Liu, Optik 182, 227 (2019).

[8] Q. Zhou, M. Ekici, A. Sonmezoglu, Optik 181, 338 (2019)..

[9] Q. Zhou, H. Rezazadeh, A. Korkmaz, M. Eslami, M. Mirzazadeh, M.R. Rezazadeh, Opt. Appl. 49, 135 (2019).

[10] A. Biswas, C.M. Khalique, Chin. J. Phys. 51, 103 (2013).

[11] A. Biswas, C.M. Khalique, Nonlin. Dynam. 63, 613 (2011).

[12] A. Biswas, M. Mirzazadeh, M. Eslami, D. Milovic, M. Belic, Frequenz 68, 525 (2014).

[13] M. Eslami, A. Neirameh, Opt. Quant. Electron. 50, 47 (2018).

[14] M. Ekici, Q. Zhou, A. Sonmezoglu, S.P. Moshokoa, M.Z. Ullah, A. Biswas, M. Belic, Superlatt. Microstruct. 107, 254 (2017).

[15] M. Inc, A.I. Aliyu, Superlatt. Microstruct. 112, 628 (2017).

[16] A.J.M. Jawad, M. Mirzazadeh, Q. Zhou, A. Biswas, Superlatt. Microstruct. 105, 1 (2017).

[17] C.M. Khalique, A. Biswas, Commun. Nonlin. Sci. Numer. Simulat. 14, 4033 (2009).

[18] E. Yasar, Y. Yildirim, Results Phys. 9, 1666 (2018).

[19] Y. Zhang, C. Yang, Weitian Yu, M. Mirzazadeh, Q. Zhou, W. Liu, Nonlin. Dynam. 94, 1351 (2018).

[20] F. Baronio, M. Onorato, S. Chen, S. Trillo, Y. Kodama, S. Wabnitz, Opt. Data Process. Storage 3, 1 (2017). 